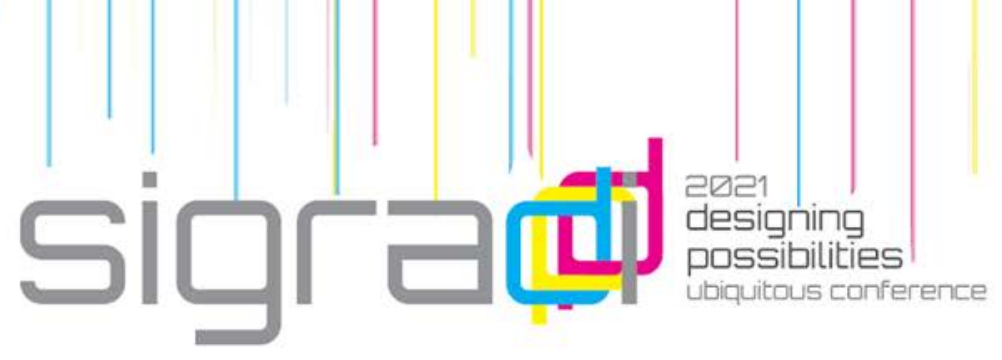

\title{
Interactive Virtual Sand Table: An Improved Alternative Participatory Design Tool for Urban Design
}

\author{
Yao Chen, Tiantian Lo, Xiangmin Guo, Xiangming Wang \\ Harbin Institute of Technology (Shenzhen), China \\ chenyaoyaoyao0809@gmail.com \\ skyduo@gmail.com \\ 24904404@qq.com \\ 1762064065@qq.com
}

\begin{abstract}
With the planning reform in China, public participation is becoming increasingly crucial to the success of rural planning. However, it is difficult to involve various stakeholders in planning and design projects, mainly due to insufficient planning knowledge and computer skills. Therefore, this paper introduces the Interactive Virtual Sand Table (IVST) as a participatory design tool so that non-professional users can use it conveniently and promote interactive and efficient collaborative design discussion. The IVST based participatory tool was applied in Xidong Village, Chaozhou City, Guangdong Province, China. The results show that IVST interaction is more natural and reduces non-professional users' difficulty participating in the design. Participants' ability to participate was highly enhanced, and their interest in IVST visualization was highly activated. In conclusion, the Interactive Virtual Sand Table highly supports the participatory village planning process and may apply to other areas and domains.
\end{abstract}

Keywords: Participatory Rural Planning; Public Participation; Interactive Virtual Sand Table; Multiplayer Collaborative Interaction; Planning And Design

\section{Introduction}

In the context of the deepening of China's urbanization process and the construction of a harmonious society in the new era, it is vital for more and more people to have the opportunity to participate in a broader range of social activities and play an increasingly crucial coordinating role. However, the traditional planning and design methods are all "top-down, expert-completed" design mode, little or no consideration of the interests of the local people, nor 
the opinions and suggestions of the local people, the majority of people have no right to participate in the planning and design. Without the participation of the masses, who are the primary users of urban planning, the planning and design cannot truly reflect the wishes and needs of most users (Zhu,2017). Participatory design is an effective means to improve urban planning (Chowdhury \& Schnabel,2020). Various methods and tools are applied to participatory planning, such as surveys, meetings, and mapping. With the development of geographic Information systems (GISs), public participation in Geographic Information Systems (PPGIS) aims to use GIS to expand public participation in decision-making (Brown \& Kytta,2014). Some innovative technologies, such as immersive geographic visualization, augmented reality, and gaming, have been around since the 2000s (Wolf, Sobke \& Wehking,2020), are receiving increasing academic interest and are being applied to the planning field.

However, there is still a gap between the need for public interaction in participatory design and the capabilities of current participatory design tools. Participatory design is not popular in China, especially in rural planning. One feature of village planning and design is that it requires more local people to be involved in the planning process. Residents are direct stakeholders and the best local knowledge (He, 2020). The problem is that most of the local population does not have the relevant expertise or the ability to manipulate complex tools. It is difficult for residents to achieve two-way communication with designers. The involvement of residents and other stakeholders is essential to prevent villages from becoming the same and losing their unique character.

Therefore, to address these challenges and needs of participatory rural planning in China, this study constructs a virtual interactive sand Table (IVST) based on virtual reality technology. This paper introduces the framework and solution of a system development technology. In addition, we also discuss the planning method of virtual interactive sand Table (IVST) based on virtual reality technology in participatory rules.

\section{Related Technologies}

Public participation, based on the belief that people affected by decisions have the right to participate in the decision-making process, has been the focus of spatial planning research and practice for decades (Jiang et al., 2018). Recently, urban design has evolved from a "top-down" to a "bottom-up" model. A variety of methods and tools have been used to achieve effective public participation.

In the earliest days, text and physical model presentations were popular methods of public participation in urban design (Dong et al., 2021). However, this approach has limited capabilities in data interpretation and spatial analysis. 
With advances in computers and information technology, computer-aided design (CAD) tools have been widely used to support the planning process by quickly generating sketches or digital planning maps for visualization and discussion (Shen et al., 2016). In recent years, traditional grid navigation systems have limited their use due to their inherent complexity and professional nature (Barton et al., 2005). Therefore, participatory GIS(PGIS) was subsequently proposed to narrow the gap between participatory planning and GIS using a simplified graphical user interface (GUI) (Mekonnen \& Gorsevski, 2015). The implementation of PGIS is a Web-based framework (Web-PGIS) that allows more non-professionals to participate in the spatial planning process and express their views anonymously (Lafrance et al., 2019). Design and analysis tools such as CAD and GIS developed for single-user professionals have limited interactive capabilities (primarily mouse, keyboard, and screen). Subsequent design and technology pioneers developed alternative interfaces, facilitating a more collaborative process of urban design. In the early 2000s, many projects developed tangible user interfaces (TUIs) (Noyman et al., 2018) for real-time creation and collaboration. They enhanced urban planning workbenches where 2D drawings, 3D physical models, and digital simulations are overlaid into a single information space to support the urban design process (Ishii et al., 2002)—introducing clay and sand into the digital design - continuous tangible user interfaces (Ishii et al., 2004). CityScope (Alonso et al., 2018) promotes consensus-building through participatory processes. Augmented reality augmented city models to show competing designs instead of outdated physical designs (Tomkins \& Lange, 2020). Some innovative technologies, such as immersive geographic visualization, augmented reality, and gaming, but their practical application in spatial planning remains exceptional or experimental (Babelon, 2021). At present, in multi-user collaborative urban planning and design (Brisco et al., 2020), the interactive way for nonprofessionals to participate in the plan is still limited. However, participatory rural planning requires face-to-face interaction rather than the online model used in urban planning. Local rural residents in China are often unfamiliar with computers and have little planning knowledge.

Therefore, many individual needs and conflicts need to be expressed, discussed, and resolved in the field. There is a need to build a participatory 3D platform that supports multi-person simultaneous communication. 


\section{$3 \quad$ Materials and Methods}

\subsection{The Requirements for IVST from Participatory Rural Planning}

The purpose of this study is to provide an easy-to-operate and multi-person collaborative IVST to improve participatory rural planning and design. IVST provides compelling visualizations and simple interactions (gestures/handles). We have chosen Three-dimensional (3D) visualization and simple interaction because 3D visualization and interaction can help them better understand design and communicate with planners. Using typical workflows of participatory planning, we can obtain specific IVST characteristic requirements(Figure.1).

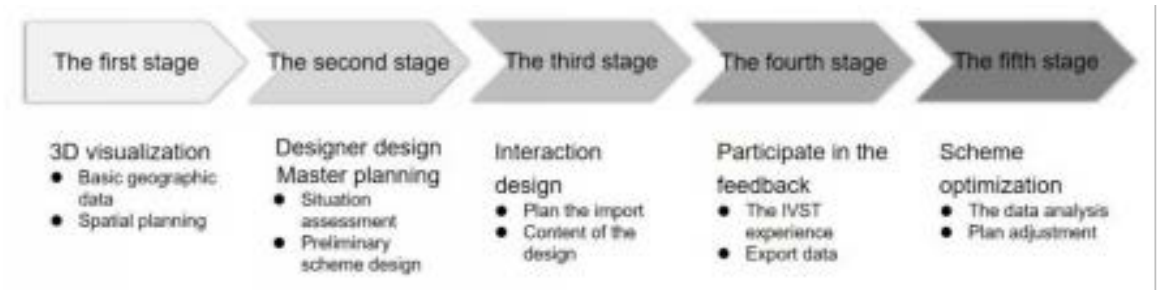

Figure 1. IVST's participatory planning workflow. The author from the drawing.

Stage 1 is planning preparation, in which primary data and information are collected about the area, such as primary geographic data, past spatial planning, natural human resources data. At this stage, IVST serves as a 3D visualization tool to support creating a current and historical 3D landscape of the area, which is the basis for further planning. Stage 2, the main tasks include state analysis (upper planning) proposed by professional designers, status assessment of designers, issuance of design assignments, and preliminary design of designers. The purpose of the second stage is to prepare necessary materials for the discussion of the next stage. Stage 3, the public interactively uses IVST for planning and design, and various stakeholders can interact directly and simultaneously in a 3D environment. Therefore, interaction design and design tools, markup, and highlighting tools are needed. Achieve effective communication between participants; this phase requires these tools to be very easy to use, enabling planners to respond quickly to shareholder requests and suggestions. Stages 4 and 5 are the later stages. The background analyzes public experience data, and the designer modifies the design scheme according to the positive feedback of stakeholders.

\subsection{General Architecture for IVST}

IVST is a virtual desktop display that allows users to modify or personalize designs in real-time and collect and analyze data on users' preferences. In this 


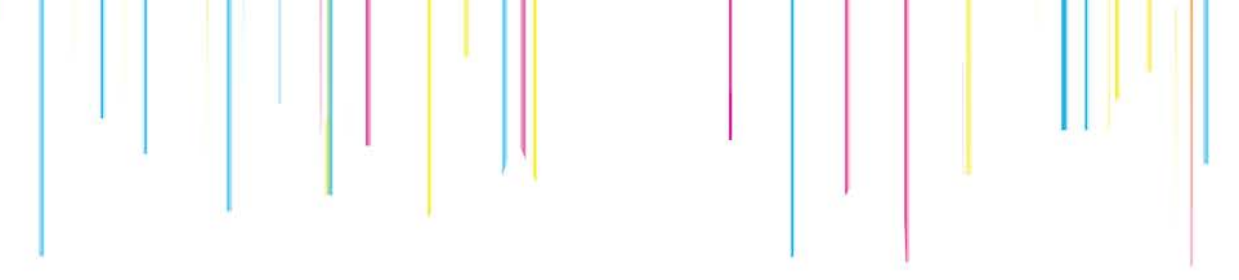

3). IVST constitutes a set of interrelated and simple participation planning tools. It allows non-professionals to create and edit 3D landscape elements interactively and quickly.

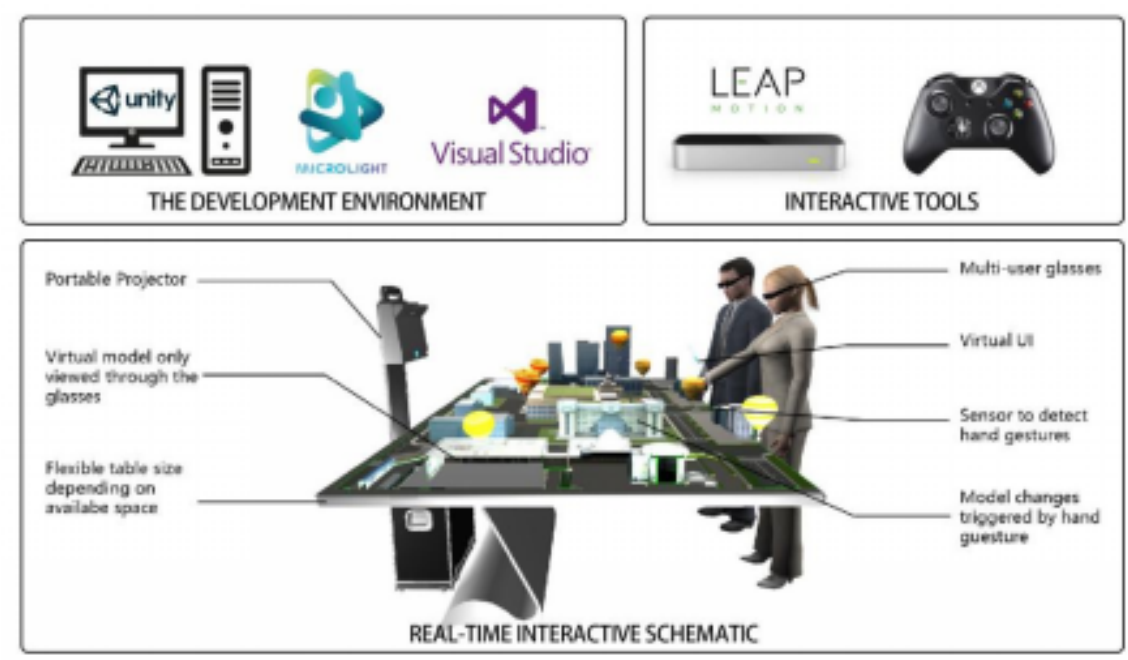

Figure 3. IVST interface schematic.Source:Yao Chen,2021.

\subsubsection{Interaction content}

This study proposes an exciting and straightforward method to study public participation in design by identifying specific design tasks and activities to explore public participation and creativity in the plan (Rushton \& Corrigan, 2021). as a game, participants wearing glasses can see a "game map," a bird's eye view of the planned area. Participants were assigned different design tasks and activities. The design task is to design buildings, roads, and plants for the designated area. Participants can select a preset component or customize them through scaling or rotating. Roads are available in three sizes. Participants also place buildings, roads, and plants in designated areas. IVST will score these positions to determine whether the task is passed or not. If the building is placed in a lousy job, an audio and text explanation will appear. Participants learn planning knowledge through scores and task ratings and unconsciously participate in the design as a game IVST reduces the difficulty of participation and engages in the design in a way that non-specialists.

For designers, it is using quantitative and qualitative analysis to achieve research objectives. The IVST will record and classify participants' preferred styles, preferred building locations, and planned developments and modifications on behalf of the researchers. Easily track and evaluate the transition steps of decision rationale and reasoning. At the same time, IVST will 
export its own recorded design activity text, easy for designers to analyze the data.

\section{Experimental System and Case Study}

This study takes the agricultural planning of Xidong Cooperative in Xidong Village, Chaoan District, Chaozhou City, Guangdong Province, China, as an example to test the feasibility of this system in rural participatory planning. Xidong Planting and Breeding Professional Cooperative are located in Chaozhou Chaoan District. By 2020, the cooperative will develop leisure agriculture and become a local web celebrity clocking point by planting flower sea, creating vegetable displays, and fruit picking. During the Spring Festival in 2021 , nearly ten thousand people will visit every day.

The main objective of this planning project is to improve the livable environment of the site by improving infrastructure, public service facilities, villagers' living conditions, and environmental sanitation. At present, the proportion difference between the surging population and the surrounding supporting facilities is too significant, so it is urgent to plan supporting public service facilities. Furthermore, plants have a growth cycle in the process of planting flower sea and developing leisure agriculture. How to calculate the reasonable cycle and grow the plants we like is a complex problem. Therefore, in the regional planning project, a participatory planning method of IVST solve the above issues.

We invited 20 volunteers to act as stakeholders as participants in this experiment. The volunteers were divided into two groups. The first group consisted of 15 non-professionals with no relevant professional knowledge and were unfamiliar with relevant software operations. The second group consists of 5 people with relevant professional expertise and a certain amount of contact time with appropriate participatory tools. These two groups of people need to design this project. The second group will analyze and create the information of the first stage after getting the tip of the first stage and provide alternative schemes for the candidate sites of the new public space. After that, the two groups engage in arbitrary discussions. There were two rounds of discussion, with only 3 participants in the first round and 17 participants in the second round. In the first round of discussion, participants expressed their opinions clearly by clicking gestures. In the second round of discussion, we added two large electronic display screens. We only set up one main console where participants take turns controlling. In addition to gestures, participants control virtual objects with a handle. Participants can also exchange ideas face-to-face on-site. And the designer analyzes the real-time exported IVST data and responds to the participants' opinions on time. Sites of choice for new public spaces proposed by designers and participants were marked and evaluated 
during several rounds of discussion at the IVST. Finally, we designed a new public space according to the appropriate location.

\section{$5 \quad$ Results}

This section begins with a summary of the workshop. We chose to present the results using the main subject categories in the data :(1) participatory design using the IVST tool;(2) Support for better understanding, creativity, collaboration, and participation. Group 1 conclusion: During the workshop, participants learned that using IVST was more like a fun placement game than a serious task, that it was easier to use gestures and clicks, and that collaboration simultaneously improved efficiency and creativity. Group 2: The designers were aware of the site's large size during the design and suggested five areas for the procedure. During the workshop, we will discuss each region in categories. While the scoring mechanism is suitable for discussions without designer participation, it is suggested to design two sets of presentation mechanisms, one for user participation and one for discussion(Figure. 4).

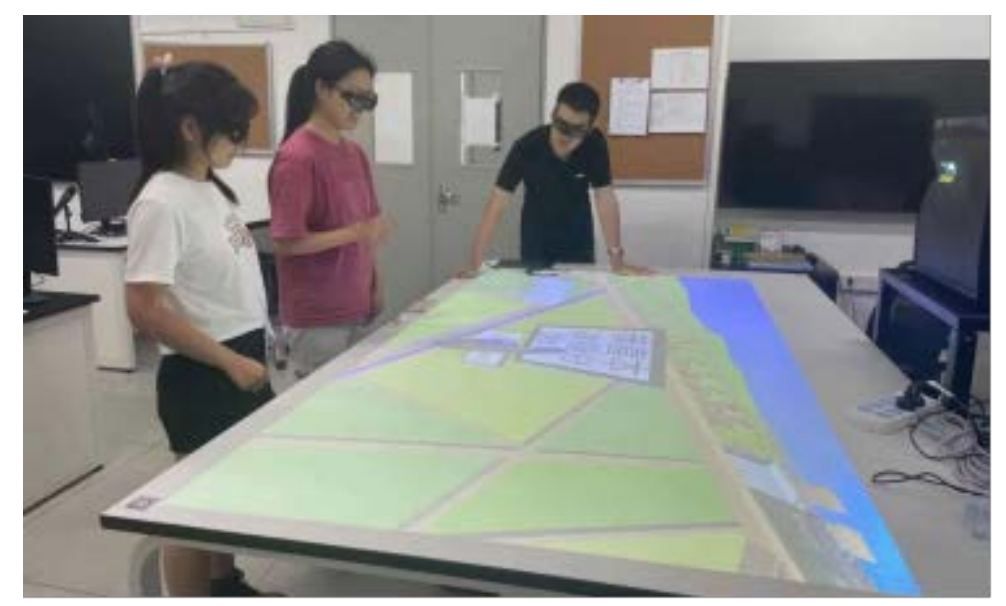

Figure 4. IVST on-site discussion experiment.Source:Yao Chen,2021.

\section{Discussion}

Gathering the will of local people is the key to successful village planning. Local villagers know most of the local knowledge (Yu et al.,2020); however, their computer knowledge is limited, and few can skillfully use these professional tools. Our experience with applications shows that non-professionals prefer simple interactions, such as grabbing or clicking objects with their hands. They 
is also applied in Xidong Village, Chaoan District, Chaozhou City, Guangdong Province, China. IVST provides a platform for multiplayer interactive 3D participation for various stakeholders to be involved in the planning. With IVST's visualization of rural planning projects, multiple stakeholders, including local villagers who know little about computers and planning, can participate in interactive planning processes quickly and smoothly with the help of the system. By exporting real-time data from the background, planners can analyze the planning scenario in real time and evaluate or modify the scheme. Our findings suggest that the quality of 3D visualization is not their focus. Ease of use and improved multi-person identification of IVST is one of the significant issues of participatory planning. However, IVST has created a new challenge that we must be addressed in the future. While we focused our attention in this study on live interaction and communication between different stakeholders, IVST cannot currently test the remote participation of those stakeholders who cannot participate in face-to-face interviews and discussions. Therefore, IVST allowing stakeholders to participate remotely and simultaneously in technology identification will focus on our future work.

Acknowledgements. This research was supported by the following funds: Later Funded Projects of National Philosophy and Social Science Foundation of China (No. 19FXWB026); Youth Project of National Natural Science Foundation of China (No. 51908158); Higher Education Research and Reform Project of Guangdong Province (No. HITSZERP19001); General Project of Stabilization Support Program of Shenzhen Universities (No. GXWD20201230155427003-20200822174038001); Science and Technology Innovation Project of Ministry of Culture and Tourism of China.

\section{References}

Alonso, L., Zhang, Y. R., Grignard, A., Noyman, A., Sakai, Y., ElKatsha, M., ... \& Larson, K. (2018, July). Cityscope: a data-driven interactive simulation tool for urban design. Use case volpe. In International conference on complex systems (pp. 253-261). Springer, Cham.

Babelon, I. (2021). Digital participatory platforms in urban planning. University of Northumbria at Newcastle (United Kingdom).

Barton, J., Plume, J., \& Parolin, B. (2005). Public participation in a spatial decision support system for public housing. Computers, Environment and Urban Systems, 29(6), 630-652.

Brisco, R., Whitfield, R. I., \& Grierson, H. (2020). A novel systematic method to evaluate computer-supported collaborative design technologies. Research in Engineering Design, 31(1), 53-81.

Brown, G., \& Kyttä, M. (2014). Key issues and research priorities for public participation GIS (PPGIS): A synthesis based on empirical research. Applied geography, 46, 122-136. 
Zheng, G. \& Liu, W. (2017). Interest balance and Knowledge Integration: the logic and model of public participation in urban planning.Doi :CNKI:SUN:ZXGL.0.2017-11010 .

Zhu Lei. (2017). Application of Participatory Approach in Landscape Planning and Design (Master's Thesis, Chinese Academy of Forestry). 